\title{
RESILIENCE ASSESSMENT OF INTERDEPENDENT CRITICAL INFRASTRUCTURE SYSTEMS
}

\author{
Quan $\mathrm{Mao}^{1}$, and $\mathrm{Nan} \mathrm{Li}^{2}$
}

\begin{abstract}
The resilience of critical infrastructure systems (CISs), in terms of the containment of spreading of system failures and function losses, and the effectiveness of post-disturbance restoration, is largely determined by how the CISs are interconnected and their interactions with each other. Despite the wide recognition of their significance in the literature, the impact of the interdependencies on the resilience of the CISs has rarely been quantitatively assessed, and the mechanism of such impact has largely remained unknown. This study models CISs interdependencies with two typical networkbased models, simulates the destruction and restoration stages of the interconnected CISs under various assumptions and settings, and based on the simulation results assesses the level of resilience of the CISs with two typical resilience metrics. A case study was conducted in a middle-sized city in China. The results showed that the resilience of the CISs may be overestimated by more than $20 \%$ if interdependencies of the CISs are not factored in. The findings suggested that to conduct comprehensive damage estimation of extreme events and optimize the efficiency of post-event restoration tasks, it is critical to consider the interdependencies of CISs and appropriately account for their impact.
\end{abstract}

Keywords: critical infrastructure, interdependency, resilience, resilience metric, cascading failure, restoration

\section{INTRODUCTION}

Critical infrastructure systems (CISs), such as electric power and water supply systems, are responsible for delivering goods and providing essential service that are fundamental to various functionalities of urban systems. According to the President's Commission on Critical Infrastructure Protection (PCCIP) (1997), CIS is defined as 'a network of independent, mostly privately-owned, manmade systems and processes that function collaboratively and synergistically to produce and distribute a continuous flow of essential goods and services'. The ever increasing interdependencies between various CISs, while improving the capacities of these interconnected systems, have also led to significant complexities in the way these systems respond to various disturbances (Ouyang 2014, Satumtira and Duenas-Osorio 2010). For instance, outages in power systems can cause subsequent failures of traffic signals and water supply pumping stations, and disturbances on telecommunication services can jeopardize the supervisory control of electric power and water supply systems. The interdependencies also play a significant role in the post-disturbance restoration of networked CISs as restoration efforts of an infrastructure component are impacted by restoration tasks

1 Graduate Student, Department of Construction Management, Tsinghua University, Beijing, China, maoq15@mails.tsinghua.edu.cn

2 Associate Professor, Department of Construction Management, Tsinghua University, Beijing, China, nanli@tsinghua.edu.cn 
in other infrastructure components and infrastructure systems (Sharkey et al 2016), and the sequence of restoration tasks needs to be scheduled accordingly to achieve optimal efficiency.

The concept of resilience, referring to the ability of a system to maintain its performance, provides a new perspective to examine the performance of CISs in case of extreme events (Ouyang and Duenas-Osorio 2012). Generally speaking, resilience refers to the ability of a system to resist, absorb and recover from a disturbance. The resilience of CISs, in terms of the containment of spreading of system failures and function losses, and the effectiveness of postdisturbance restoration, is largely determined by how the CISs are interconnected and their interactions with each other. Despite wide recognition of the significance of the interdependencies in the literature, which has led to an increasing volume of research that aims to model networked CISs and their interdependencies, the impact of the interdependencies on the resilience of the CISs has rarely been quantitatively assessed, and the mechanism of such impact has largely remained unknown. This paper aims to address this gap, by assessing the impact of interdependencies on the resilience of CISs. Two typical network-based models are selected from the literature for modeling interdependent CISs, and two resilience metrics are selected for assessing the level of resilience of these CISs against certain simulated extreme events. The results are expected to provide insight on the impact of interdependencies on CISs, and support future decision making of CIS protection against possible extreme events and consequent cascading failures.

\section{SELEcted Models AND Metrics}

A number of different models has been developed in prior research for modeling CISs interdependencies. These models can be broadly categorized as agent-based models, inputoutput models and network-based models (Satumtira and Duenas-Osorio 2010, Ouyang 2014). Network-based models are increasingly adopted in recent studies due to their capability of visually describing the details of interdependencies and analyzing disturbance propagations at the component level (Ouyang 2014). CISs can be modeled as networks, where nodes represent built facilities, such as water plants and electric substations, and links represent relational connections, such as transmission lines and pipelines, among the nodes. Networkbased models can be generally grouped into topology-based models and flow-based models based on Ouyang (2014)'s review. For these two groups of models, the most representative models are the ones proposed by Duenas-Osorio et al. (2007) (hereinafter referred to as Model 1) and Lee et al. (2007) (hereinafter referred to as Model 2), respectively. Researchers have proposed different metrics, such as network connectivity, system performance, the population loss and economic loss, to measure system performance and assess system resilience. Among these metrics, the ones proposed by Ouyang and Duenas-Osorio (2012) (hereinafter referred to as Metric 1) and Reed et al. (2009) (hereinafter referred to as Metric 2) are the most representative and effective, as have been widely used in prior research. Therefore, Models 1 and 2, and Metrics 1 and 2 are selected in this study to model CISs and assess the impact of interdependencies among them on their overall level of resilience. These models and metrics are further explained below.

Model 1 is a probabilistic topological network model. It was proposed by Duenas-Osorio et al. (2007) to simulate the interdependencies between electric power and water supply systems. Given limited knowledge of coupling details between components of these two 
systems, the researchers proposed that the failure of a component is conditional, at a certain probability, to the failure of components that it relies on to function. For instance, in reality a failed component in the electric power supplies system could cause the failure of interconnected components in the water supplies system at a certain probability, and vice versa. Based on empirical data it was also found that closer proximity of the interconnected components could result in lager probability of the cascading failure. Therefore, the conditional probability of cascading failure between two components could be determined based on their spatial proximity.

Model 2 is a flow network model. It was proposed by Lee et al. (2007) to simulate the interdependencies between electric power, telecommunications and subway systems. In this model, each node or link has two parameters, including flow and capacity. Flow refers to the actual rate at which commodities pass through a given node or link, and capacity refers to the maximum flow a given node or link allows. If component $i$ in one CIS relies on goods or service from component $j$ from another CIS to function, the interruption of this goods or service can reduce the capacity of $i$. It is assumed in this model that the flow cannot exceed its capacity, and that the flow supply must be equal to flow demand in the same CIS. The flow redistribution in CISs, which aims at satisfying as much demands as possible right after a disturbance by changing and rebalancing the flow of each node or link without exceeding the capacities, is also considered in this model.

Both selected resilience metrics are calculated as the integral of the ratio of actual level of system performance over the initial or desired level of performance over the entire disturbance event cycle, including the destruction stage and the restoration stage. Both metrics can be expressed as:

$$
=\frac{\int_{t_{0}}^{t_{1}} Q(t) d t}{Q_{0}\left(t_{1}-t_{0}\right)}
$$

where $R$ is the level of resilience, $Q_{0}$ is initial level of system performance, $Q(t)$ is remaining level of system performance after disturbance happens, $t_{0}, t_{1}$ are the time of disturbance occurrence and system full recovery. The two selected resilience metrics differ mainly in terms of how they define system performance. Metric 1 defines system performance as the ratio of current flow in the system at a given point of time in the disturbance event cycle over the flow when system is under a normal state (Ouyang and Duenas-Osorio 2012). Metric 2 defines system performance as the percentage of satisfied demand relative to the total demand, which is usually represented by the number of customers the system serves. It is another popular performance indicator as it to some extent reflects the social impact of the system (Reed et al 2009).

\section{Model IMPlementaton AND REsilience AsSESSMENT}

To simulate the destruction and restoration stages of the interdependent CISs, this study implemented the above two models, and simulated the responses of three networked CISs to a regional disturbance caused by flooding in a case city. The implementation of the models was based on descriptions of these models in (Duenas-Osorio et al 2007, Lee et al 2007), as briefly summarized in the last section, in terms of assumptions, model structure, parameter 
settings and procedures. Where certain model details were not available in prior studies, additional reasonable assumptions were made. The case city settings, model implementation, and assessment of the resilience of interdependent CISs, are explained in detail below.

\subsection{Case City}

A case study was conducted in a middle-sized city in China in this paper, where three CISs, including the electric power supplies, water supplies and telecommunication, were examined. The CISs components, their capacities, and connections among them, as well as normal flow quantities and service demands, were collected from CISs design and operation schemes provided by local authorities, and the flow information was collected from system monitoring data and historical records. Due to the incompletion of real data needed for developing the network models, certain interdependencies that were not documented in available design schemes were set according to common sense. For instance, nodes in water supplies and telecommunication systems require power supplies from nearby nodes in the electric power supplies to function, and nodes in water supplies and electric power supplies systems rely on nodes in the telecommunication systems for remote sensing and control.

\subsection{Damage and Restoration}

A regional disturbance, instead of a nodal disturbance, was simulated in the case city, in order to expose the CISs models to a possible scenario where multiple nodes are damaged and require restoration, the sequence of which needs to be optimized in a procedure that is impacted by the interdependencies of the CISs. Flooding was determined to be the most likely regional disturbance in the case city based on its history. One area near the city center was selected due to high probability of flooding and the importance of CISs components located within this area. All CISs components in this area were assumed to lose their functions when hit by the flood and require restoration efforts to recover.

Two aforementioned network models were implemented to represent the CISs and their interdependencies, and simulate the destruction stage in the case study. Built facilities, such as water plants and electric substations, were regarded as nodes while transmission lines and pipelines were regarded as links in the network. In Model 1, interdependencies were set as a conditional probability of failure. Unlike the original model in which the conditional probability was determined by spatial proximity of the components that represents their level of dependency, this case study used flow to represent the dependency level and determine the conditional probability of failure. Specifically, it was assumed that the conditional probability of failure of node $j$ in CIS $q$ caused by failure of node $i$ in CIS $p$ was calculated as:

$$
P_{i \rightarrow j}=\frac{F_{i \rightarrow j}}{\sum_{r}^{p} F_{r \rightarrow j}}
$$

where $P_{i \rightarrow j}$ refers to the conditional probability of failure of node $j$ resulted from failure of

node $i, F_{i \rightarrow j}$ refers to the flow from $i$ to $j, \sum_{r}^{p} F_{r \rightarrow j}$ refers to the total amount of flow from CIS $p$ to node $j$. One exception is the conditional probability of cascading failure caused by information interdependency, namely the reliance of CIS components on telecommunication services for remote sensing and control. It is set as a constant of 0.2 based on Dudenhoeffer et al.'s work (2006), because this type of interdependency mainly affects the flow 
redistribution capability of the CISs instead of their performance, and its impact is not determined by the flow.

For Model 2, following Lee at al.'s work (2007), the normal flow of the CISs components was determined by historical monthly flow, and the normal capacity was assumed as 1.2 times of the normal flow based on Ouyang and Duenas-Osorio's work (2012). In addition to the original model, it was assumed in this case study that the node of electric power supplies or water supplies would not be able to conduct redistribution or change its flow due to loss of remote sensing control functions when the telecommunication nodes it relies on are damaged.

Interdependencies are an important factor to consider in determining the restoration priority of damaged nodes. The delivery contribution of a node, which is defined as the ratio of the flow the node delivers over the total flow in the system, was selected as an assessment of the priority. When considering interdependencies in the restoration priority assessment, the delivery contribution should be re-assessed by taking into account the nodes' contribution to other CISs. For instance, if node $j$ in CIS $q$ relies on node $i$ in CIS $p$ to function, the delivery contribution of node $i$ should include the flow form node $i$ to node $j$.

During the restoration stage, restoration resources were assumed to be limited. Therefore, in any given CIS up to one node could be under restoration at any given point of time. Each unit of time was set as $T$ in the simulation and it was assumed that a failed node could recover to normal state after $5 T$ since restoration of the node started. In Model 1, since the state of each node was binary, a failed node would return to normal state after 5T. In Model 2, the performance curve of a failed node during the restoration stage was set as non-linear since the initial restoration was faster due to higher level of preparedness in man-made systems (Kafali and Grigoriu 2005). Specifically, the performance level of the node was set 0.4, 0.65, $0.8,0.9$ and 1.0at $T, 2 T, 3 T, 4 T$ and $5 T$, respectively.

\subsection{Resilience Metrics}

Metric 1and Metric 2 were selected to measure the system performance of CISs in this study. For Metric 1, the amount of the flow each node delivered to end users was identified by the difference between in-flow and out-flow of the node. For Metric 2, the number of customers each node served was identified based on the population of administrative regions each node served. Based on simulation results, the level of resilience was calculated based on Equation (1). For either metric, the assessed resilience of three different CISs was averaged to calculate the overall resilience of the entire networked system.

\section{FINDINGS}

To assess the impact of interdependencies on the resilience of interdependent CISs, simulations were carried out under and compared between three different assumptions, including not considering interdependencies in destruction or restoration (Simulation assumption 1), considering interdependencies in destruction only (Simulation assumption 2), and considering interdependencies in both destruction and restoration (Simulation assumption 3). Four simulation settings were applied to each of the three situations. These settings included (a) simulated with Model 1, assessed with Metric 1; (b) simulated with Model 2, assessed with Metric 1; (c) simulated with Model 1, assessed with Metric 2; and (d) simulated with Model 2, assessed with Metric 2. Based on the model implementation and resilience 
assessment process explained in the last section, the responses of the CISs to a flooding event in the case study were simulated, and the results are depicted in Figure 1.

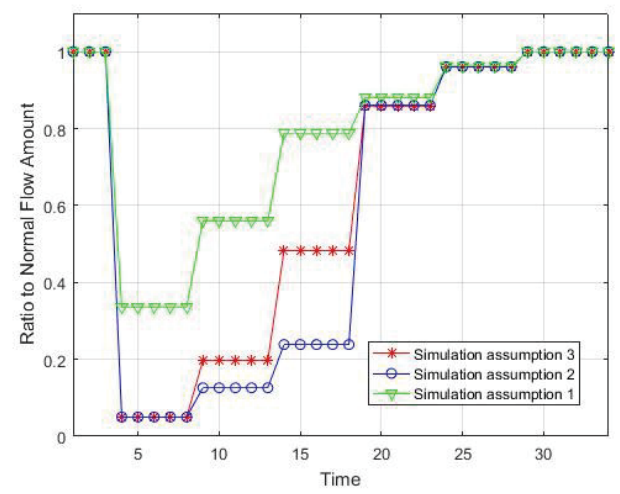

Simulation setting a

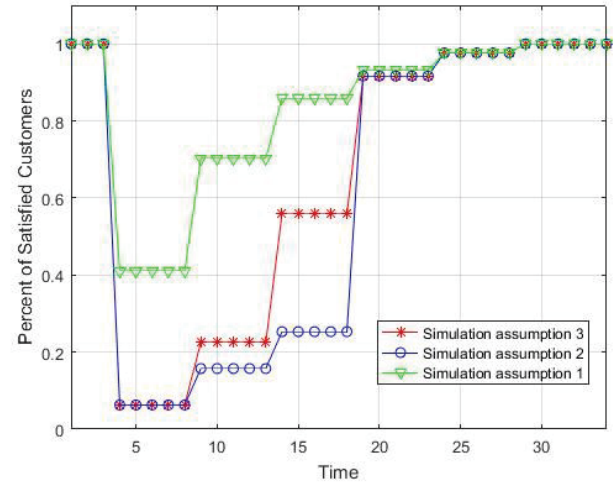

Simulation setting c

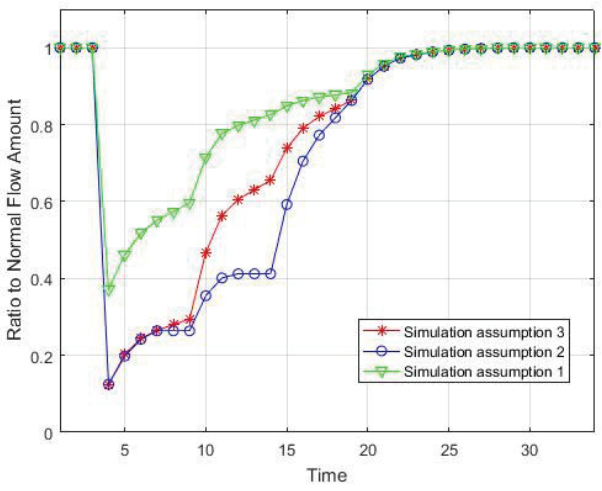

Simulation setting $b$

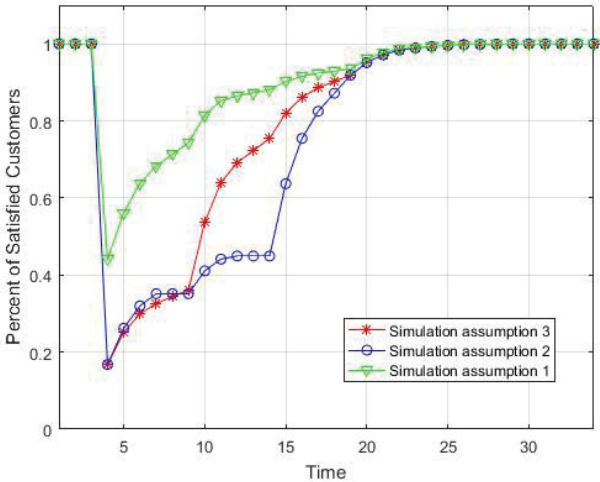

Simulation setting $d$

Figure 1: Simulation results of the response of CISs to a flooding event under different simulation assumptions and simulation settings

Comparing results under simulation assumption 1 and those under Simulation assumption 2, it could be seen that the decrease in the performance was noticeably significant when interdependencies and the resulting cascading failures were considered during the destruction stage. The magnitude of the performance loss was $43.1 \%, 39.3 \%, 59.1 \%$ and $48.9 \%$ higher, when interdependencies were considered, under simulation settings a, b, c and d, respectively.

Comparing results under simulation assumption 2 and those under simulation assumption 3 , it could be seen that a faster recovery in system performance occurred in the restoration stage when interdependencies were considered. The speed of performance recovery was $14.0 \%$, $8.2 \%, 15.9 \%$ and $8.8 \%$ higher, when the restoration sequence was optimized with interdependencies taken into consideration, under simulation settings $\mathrm{a}, \mathrm{b}, \mathrm{c}$ and $\mathrm{d}$, respectively.

The overall level of resilience of the networked CISs under different simulation assumptions and simulation settings were calculated, and summarized in Table 1 . The results showed that, in general, the level of resilience was $21.2 \%$ and $22.1 \%$ lower in the case study, 
when interdependencies were considered, based on Metric 1 and Metric 2, respectively. Specifically, taking the interdependencies into account resulted in decrease of resilience in the destruction stage and increase of resilience in the restoration stage.

Table 1: Resilience assessment in different simulation assumptions.

\begin{tabular}{cccccc}
\hline & & \multicolumn{5}{c}{ Simulation Setting } \\
& & $($ a) & $($ b) & (c) & (d) \\
\hline \multirow{3}{*}{ Simulation } & 1 & 0.705 & 0.807 & 0.776 & 0.863 \\
Assumption & 2 & 0.447 & 0.636 & 0.473 & 0.676 \\
& 3 & 0.510 & 0.688 & 0.548 & 0.735 \\
\hline
\end{tabular}

\section{CONCLUSIONS}

This study simulated the destruction and restoration stages of CISs in response to a regional disturbance using two network-based models, and assessed the impact of interdependencies on the resilience of CISs in a quantitative way using two metrics. The simulation results showed that, regardless of the network-based CIS model and CIS resilience metric, the interdependencies between different CISs had significant impact on the response of the networked CISs to simulated extreme events during the destruction and restoration stages and their overall level of resilience. Specifically, the results showed that the vulnerability of CISs to extreme events and resources and efforts required to require them would be significantly underestimated if interdependencies of the CISs were not factored in, leading to misleading estimation of the overall level of resilience of the CISs. The findings suggested that to conduct comprehensive damage estimation of extreme events and optimize the efficiency of post-event restoration tasks, it is critical to consider the interdependencies of CISs and appropriately account for their impact. It needs to be noted that the approach described in this paper is applicable to CISs which have network type of topology, are well-networked, and whose flows can be measured and recorded. Extending this approach to other types of critical infrastructures would require further research. Data availability is another major challenge that may benefit from additional data sources such as CISs sensing data and simulation data, which can be investigated in the future.

\section{ACKNOWLEDGMENTS}

This material is based upon work supported by Tsinghua University Initiative Scientific Research Program under Grants No. 2014z21050 and 2015THZ0. The authors are thankful for the support of Tsinghua University. Any opinions, findings, and conclusions or recommendations expressed in this paper are those of the authors and do not necessarily reflect the views of Tsinghua University. 


\section{REFERENCES}

Dudenhoeffer, DD., Permann, MR. and Manic, M. (2006). CIMS: A Framework for Infrastructure Interdependency Modeling and Analysis. In: 2006 Winter Simulation Conference. [online] Monterey: IEEE, pp. 478-485. Available at: http://ieeexplore.ieee.org/document/4117643/ [Accessed 05 Mar. 2007]

Duenas-Osorio, L., Craig, J. and Goodno, B. (2007). Seismic Response of Critical Interdependent Networks, Earthquake Engineering \& Structure Dynamics, 36(2), pp. 285306.

Kafali, C. and Grigoriu, M. (2005). Rehabilitation Decision Analysis, In: the Ninth International Conference on Structural Safety and Reliability.

Lee II, E.E., Mitchell, J.E. and Wallace, W.A. (2007). Restoration of Services in Interdependent Infrastructure Systems: A Network Flows Approach, IEEE Transactions on Systems, Man and Cybernetics Part C: Applications and Reviews, 37(6), pp. 1303-1317.

Ouyang, M. (2014). Review on Modeling and Simulation of Interdependent Critical Infrastructure Systems, Reliability Engineering \& System Safety, 121, pp. 43-60.

Ouyang, M. and Duenas-Osorio, L. (2012). Time-dependent Resilience Assessment and Improvement of Urban Infrastructure Systems. Chaos: An Interdisciplinary Journal of Nonlinear Science, 22(3), p.033122.

President's Commission on Critical Infrastructure Protection (PCCIP) (1997). Critical Foundations: Protecting America's Infrastructures: the Report of the President's Commission on Critical Infrastructure Protection, U.S. Government Printing Office, Washington, D.C.

Reed, D.A., Kapur, K.C. and Christie, R.D. (2009). Methodology for Assessing the Resilience of Networked Infrastructure, IEEE Systems Journal, 3(2), pp. 174-180.

Satumtira, G. and Duenas-Osorio, L. (2010). Synthesis of Modeling and Simulation Methods on Critical Infrastructure Interdependencies Research. In: K. Gopalakrishnan \& S. Peeta, ed., Sustainable Infrastructure Systems: Simulation, Imaging, and Intelligent Engineering, $1^{\text {st }}$ ed. New York: Springer Berlin Heidelberg, pp. 1-51.

Sharkey, T., Nurre, S., Nguyen, H., Chow, J., Mitchell, J. and Wallace, W. (2016). Identification and Classification of Restoration Interdependencies in the Wake of Hurricane Sandy, Journal of Infrastructure Systems, 22(1), pp. 04015007. 